\title{
Anti-amoebic potential of azole scaffolds and nanoparticles against pathogenic Acanthamoeba
}

\author{
Shweta Walvekar ${ }^{\mathrm{a}}$, Ayaz Anwar ${ }^{\mathrm{a}, *}$, Areeba Anwar $^{\mathrm{b}}$, Nanthini Sridewi ${ }^{\mathrm{b}}$, Mohammad Khalid ${ }^{\mathrm{c}}$, \\ Yoon Yen Yow ${ }^{\mathrm{a}}$, Naveed Ahmed Khan ${ }^{\mathrm{d}}$ \\ ${ }^{a}$ Department of Biological Sciences, School of Science and Technology, Sunway University, Subang Jaya 47500, Selangor, Malaysia \\ ${ }^{\mathrm{b}}$ Faculty of Defence Science and Technology, National Defence University of Malaysia, 57000 Kuala Lampur, Malaysia

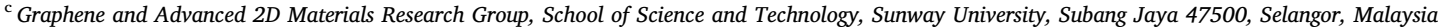 \\ ${ }^{\mathrm{d}}$ Department of Biology, Chemistry and Environmental Sciences, College of Arts and Sciences, American University of Sharjah, Sharjah 26666, United Arab Emirates
}

\section{A R T I C L E I N F O}

\section{Keywords:}

Azole

Free-living amoeba

Acanthamoeba

Anti-amoebic

Ergosterol pathway

\begin{abstract}
A B S T R A C T
Acanthamoeba spp. are free living amoeba (FLA) which are widely distributed in nature. They are opportunistic parasites and can cause severe infections to the eye, skin and central nervous system. The advances in drug discovery and modifications in the chemotherapeutic agents have shown little improvement in morbidity and mortality rates associated with Acanthamoeba infections. The mechanism-based process of drug discovery depends on the molecular drug targets present in the signaling pathways in the genome. Synthetic libraries provide a platform for broad spectrum of activities due to their desired structural modifications. Azoles, originally a class of synthetic anti-fungal drugs, disrupt the fungal cell membrane by inhibiting the biosynthesis of ergosterol through the inhibition of cytochrome P450 dependent $14 \alpha$-lanosterol, a key step of the sterol pathway. Acanthamoeba and fungi share the presence of similar sterol intermediate, as ergosterol is also the major endproduct in the sterol biosynthesis in Acanthamoeba. Sterols present in the eukaryotic cell membrane are one of the most essential lipids and exhibit important structural and signaling functions. Therefore, in this review we highlight the importance of specific targeting of ergosterol present in Acanthamoebic membrane by azole compounds for amoebicidal activity. Previously, azoles have also been repurposed to report antimicrobial, antiparasitic and antibacterial properties. Moreover, by loading the azoles into nanoparticles through advanced techniques in nanotechnology, such as physical encapsulation, adsorption, or chemical conjugation, the pharmacokinetics and therapeutic index of the drugs can be significantly improved. The current review proposes an important strategy to target Acanthamoeba using synthetic libraries of azoles and their conjugated nanoparticles for the first time.
\end{abstract}

\section{Introduction}

Acanthamoeba is a free-living ameba (FLA), isolated from wide variety of environments such as soil, water supplies, swimming pools, hospitals etc. (De Jonckheere, 1991). FLA can resist to extreme conditions such as extended time of desiccation, high/low temperatures, $\mathrm{pH}$ and radiations (Khan, 2006). In addition to its natural distribution, Acanthamoeba can be opportunistically pathogenic, being identified as the causative agent of Acanthamoeba keratitis (AK) which is a painful and sight-threatening infection of the cornea, and granulomatous amoebic encephalitis (GAE) which is rare but a fatal central nervous system (CNS) infection (Culbertson et al., 1961; Jones et al., 1975). Acanthamoeba can enter the body via a break in the skin or inhalation of wind-blown cysts and may cause cutaneous, nasopharyngeal and disseminated infection and subsequently spread hematogenously to the CNS leading to GAE.

The life cycle of Acanthamoeba consists of two stages: an actively feeding, dividing trophozoite and a dormant cyst. The trophozoite stage exists in the favorable conditions (neutral $\mathrm{pH}$, availability of sources of nutrients, optimal temperature of about $30^{\circ} \mathrm{C}$ ). They feed on bacteria, yeast, algae or small organic particles and their size measures between 25 to $40 \mu \mathrm{m}$ (Khan, 2006). The double-walled wrinkled cyst is composed of an ectocyst and an endocyst ranging in size from 13 to $20 \mu \mathrm{m}$ and varies from species to species. The outer wall consists of proteins and polysaccharides, while the inner wall possesses cellulose. Cyst formation occurs under adverse environmental conditions such as food

\footnotetext{
* Correspondence author.

E-mail address: ayazanwarkk@yahoo.com (A. Anwar).
} 
deprivation, desiccation, and changes in temperature and $\mathrm{pH}$ (Dudley et al., 2009).

\subsection{Current treatment options against Acanthamoeba infections}

For the past several decades, there has been little improvement in the morbidity and mortality associated with Acanthamoeba diseases. Antimicrobial chemotherapy is the most widely used method of treating infections caused due to Acanthamoeba with various combinations of drugs such as amphotericin B, trimethoprim-sulfamethoxazole, rifampin, ketoconazole, fluconazole, sulfadiazine, miltefosine etc (Marciano-Cabral, 2003). The rate of development of novel anti-acanthamoebic chemotherapies of translational value and the lack of interest of the pharmaceutical industry in developing such chemotherapies have been disappointing (Khan et al., 2017). Hence, there is an urgent need to develop a targeted therapeutic approach to identify drugs that can affect Acanthamoeba viability without affecting the host cells.

\subsection{Acanthamoeba keratitis}

Acanthamoeba spp. are most commonly introduced to the eye by contact lenses that have been exposed to the organism through the use of contaminated lens solution, using homemade saline-based solution or tap water, or from wearing contact lenses while bathing or swimming (Lorenzo-Morales et al., 2015). Acanthamoeba keratitis (AK) is a rare disease in which amoebae invade the cornea. Clinical symptoms are often corneal pain and photophobia, which may be disproportionate to the appearance of the eye. At early stages scattered epithelial erosions, anterior stromal haze, nummular keratitis and variable stromal edema associated with keratitis precipitates on endothelium was observed in Acanthamoeba keratitis (Garg et al., 2017). A diagnosis of AK should be considered when chronic corneal ulcers are unresponsive to the antibiotic therapy. Rapid diagnosis of the disease is paramount in lowering the number of patients who require penetrating keratoplasty (Sadiq et al., 1998), which was the only form of rehabilitation. Keratoplasty was effective in eliminating infectious pathogens and also prevented the recurrence of the same infection is most cases, but in some rare cases it proved to be unsuccessful (Kumar and Lloyd, 2002).

Currently therapeutic agents have been tried in various combinations, but none of the managements have proved to be particularly effective (Schuster and Visvesvara, 2004a). Furthermore, it is also important to consider drugs that act effectively against cysts forms. Usually treatments based on biguanide $(0.02 \%$ of polyhexamethylene biguanide or $0.02 \%$ chlorhexidine digluconate) in conjunction with a diamidine $(0.1 \%$ propamidine isethionate or $0.1 \%$ hexamidine $)$ are recommended (Pérez-Santonja et al., 2003). If bacteria are also associated with the infection, addition of antibiotics, i.e., neomycin or chloramphenicol is suggested. The presence of antibiotics limits possible bacterial infection or, at the very least, eliminates the food source for Acanthamoeba. Imidazoles such as miconazole, itraconazole and ketoconazole have been used with limited success (Berger et al., 1990; D'Aversa et al., 1995; Ishibashi et al., 1990).

\subsection{Granulomatous amoebic encephalitis (GAE)}

GAE is a subacute to chronic granulomatous infection of the CNS caused by the species of Acanthamoeba., Balamuthia mandrillaris and Sappina pedate. These CNS infections are reported to occur in mostly immunocompromised individuals like post-transplantation, HIV infection etc., and occasionally in immunocompetent hosts (MarcianoCabral and Cabral, 2003; Stidd et al., 2012).

The successful treatment depends on early diagnosis of these GAE cases. Most of the cases are detected at late stages and hence high mortality has been observed. Retrospective analysis of survival cases reveals a combination of surgical resection of the affected lesion and a regimen of multiple antibiotics (Orozco et al., 2011). Current therapeutic agents include a combination of ketoconazole, fluconazole, sulfadiazine, pentamidine isethionate, amphotericin B, azithromycin, itraconazole or rifampin that may be effective against CNS infections but have severe side-effects (Schuster and Visvesvara, 2004b). Recent studies have suggested that alkyl phosphocholine compounds, such as hexadecyl phosphocholine, exhibit anti-Acanthamoeba properties as well as the ability to cross the blood-brain barrier and may thus have value in the treatment of GAE (Walochnik et al., 2002). Miltefosine, azoles, pentamidine, and cotrimoxazole were used in the treatment of $>90 \%$ successfully treated GAE cases. Recent in vitro studies show loperamide, haloperidol, apomorphine, procyclidine, and amiodarone as promising drugs that can be utilized in the treatment of GAE infections (Kulsoom et al., 2014).

\subsection{Cutaneous acanthamoebiasis}

The cutaneous infections are characterized by nodules and skin ulcerations and demonstrate Acanthamoeba trophozoites and cysts. The cutaneous infections are most common in patients with AIDS, with or without CNS involvement (DELUOI et al., 1996; Casper et al., 1999; Niederkorn, 2002). The treatment of acanthamoebiasis has not been well established and is based largely on in vitro sensitivity of the organism to several chemotherapeutic agents. Therapy is less successful when CNS involvement occurs. However, successful treatments of cutaneous acanthamoebiasis using itraconazole, pentamidine, 5-fluorocystosine, and topical chlorhexidine gluconate and ketoconazole cream have been reported (Helton et al., 1993; Slater et al., 1994).

\subsection{Azole compounds as therapeutic agent against free-living amoebae}

Azoles are basically five-member heterocyclic compounds containing one or more different hetero atom out of which at least one must be nitrogen and another like sulfur or oxygen. Synthesis of compounds incorporating five-membered heterocyclic rings have been attracting interest over the past decade because of their various applications such as propellants, explosives, pyrotechnics and chemotherapy (Chavez and Parrish, 2009). Azole heterocycles represent one of the most active classes of compounds which possess a wide spectrum of biological activities such as antibacterial, antifungal and antimicrobial activities (Anderluh et al., 2009; Çolak et al., 2010). In the field of medicinal chemistry, azoles are widely used and studied class of antimicrobial agents due to their safety profile and high therapeutic index (Ashok et al., 2007). Azole compounds with electron-rich nitrogen heterocycles play an important role in medicinal field and thus they can bind easily with the enzymes and receptors in organisms through weak interactions thereby exhibiting various bioactivities (Peng et al., 2013). Among the important pharmacophores responsible for antimicrobial activity, the azole scaffolds are considered as a viable lead structure for the synthesis of more efficient antimicrobial agents (Rostom et al., 2009).

Azoles inhibit the synthesis of sterols in fungi by inhibiting cytochrome P450-dependent $14 \alpha$-lanosterol demethylase, which removes the methyl group on C-14 of lanosterol, a key intermediate step in the formation of ergosterol in the fungal cell membrane (Bryskier, 2005). In protozoa, sterol biosynthesis pathway is absent in strict anaerobic organisms, including human pathogens, Giardia, Entamoeba, Cryptosporidium, and Trichomonas (Desmond and Gribaldo, 2009). In a variety of free-living and symbiotic protist species, some of which are important human parasites, are reported to synthesize sterols de novo. Thus, the sterol biosynthesis pathway is present in free-living amoebas like Acanthamoeba and Naegleria (Raederstorff and Rohmer, 1985, 1987; Lamb et al., 2015).

Azole compounds alter plasma membrane permeability in fungi (Bodey, 1992). Thus, the research for effective chemotherapeutic agents can be focused on those with the mechanisms of action that 
modify the plasma membrane of these eukaryotic organisms, which causes loss of essential ions and upsets water balance in the cell (Schuster, 1993).

\subsection{Anti-parasitic activity of azoles}

Although azoles are originally developed as antifungal agents, azole compounds have also been explored for activity on kinetoplastids such as Leishmania since these parasites also require ergosterol for their metabolism and share this biosynthetic pathway with fungi. Among the several drugs tested (fluconazole, itraconazole, ketoconazole), only ketoconazole was found to be consistently efficacious and is now used for the treatment of cutaneous leishmaniasis infections caused by $L$. Mexicana (Nagle et al., 2014). Imidazoles containing compounds have received considerable attention in the search for leishmaniasis chemotherapy due to the success of agents such as ketoconazole, miconazole, econazole, and clotrimazole in treating fungal infections, thus lending credence to the possible utility of this broad class of compounds in other types of infections (Nagle et al., 2014). Ketoconazole and Itraconazole have been shown to be potent antiproliferative agents against Trypanosoma cruzi, both in vitro and in vivo. Ketoconazole can eliminate T. cruzi amastigotes from a tissue culture system. At very low temperatures also, it was effective in causing changes in the sterol composition of trypomastigotes, but it did not affect the propagation or sterol composition of the human tissue host-cell (Goad et al., 1989).

For bacterial infections and pathogenic protozoan parasites, 2-methyl 5 nitro imidazole-based drugs are being used for years (Mukherjee and Boshoff, 2011; Upcroft et al., 1999). Currently, 2-methyl 5 nitroimidazole derivatives which are available in the market are tinidazole, ornidazole, secnidazole and are highly recommended for the treatment of stages of amoebiasis (Azam and Agarwal, 2007). Dioxazole, bearing oxygen and nitrogen both, also displayed significant inhibitory activity against $E$. histolytica (Bhat et al., 2009).

There are numerous anti-amoebic azole drugs used in medical practice like metronidazole and tinidazole, which kills amoeba in the host tissue and organ (Singh et al., 2009). Metronidazole is a therapeutic agent of choice for amoebiasis and is also used in combination with antimicrobial drugs against yeast infections. Under anaerobic conditions inside the cell, it is reduced to a cytotoxic nitro radical and binds non-specifically to the organism's DNA and enzymes, which are thus inactivated (Rasmussen et al., 1997). But high doses of drugs may have some severe side effects and resistance to this drug in many pathogenic bacteria and protozoa (Adagu et al., 2002). Benzimidazole and its derivatives are widely used in searches for new drugs (Craigo et al., 1999). Biological assays against $E$. histolytica indicate that, with the very few exceptions, most of the benzimidazole derivatives demonstrated higher activity than metronidazole (Singh et al., 2009).

Voriconazole has been tested against trophic stages of several clinical isolates of Acanthamoeba spp., and other free-living amoebae such as Balamuthia mandrillaris, Naegleria fowleri. Voriconazole had little or no inhibitory or amoebicidal effect upon the growth of Balamuthia amebae at all concentrations tested but was found to have a potent inhibitory effect when tested against Acanthamoeba spp. (Schuster et al., 2006). In 1993, Schuster found that the triazoles, fluconazole and itraconazole were ineffective against $A$. polyphaga, while the imidazoles bifonazole and clotrimazole were effective against all types of Acanthamoeba spp. infections (Schuster, 1993). Clotrimazole and miconazole were reported as equally effective in vitro against Naegleria (Duma and Finley, 1976). Ketoconazole was found to be as effective as amphotericin B against a clinical isolate of Naegleria but less so fluconazole and itraconazole (Tiewcharoen et al., 2002). The results of anti-amoebic activities of azole drugs against free-living amoebae are summarized in table 1.
Table 1

Comparative list of azole drugs against Acanthamoeba.

\begin{tabular}{|c|c|c|c|c|}
\hline No & Drug & Structure & Mode of action & Effects \\
\hline 1 & Voriconazole & & $\begin{array}{l}\text { Inhibits } 14 \alpha- \\
\text { demethylase, a } \\
\text { cytochrome P-450 } \\
\text { enzyme necessary to } \\
\text { convert lanosterol to } \\
\text { ergosterol (Schuster et } \\
\text { al., 2006) }\end{array}$ & \begin{tabular}{|l} 
Deteriorate \\
subcellular \\
components \\
leading to cell \\
necrosis. \\
Most effective \\
compared to \\
other azoles.
\end{tabular} \\
\hline 2 & Clotrimazole & & \begin{tabular}{|l|} 
Inhibits endogenous \\
respiration by impairing \\
triglyceride and \\
phospholipid \\
biosynthesis as well as \\
to inhibit cellular \\
calcium homeostasis \\
and calcium ATPases \\
(Schuster 1993).
\end{tabular} & $\begin{array}{l}\text { Effective against } \\
\text { Naegleria \& } \\
\text { Acanthamoeba }\end{array}$ \\
\hline 3 & Itraconazole & & \begin{tabular}{|l} 
Inhibits ergosterol \\
biosynthesis and has \\
been shown to inhibit \\
endogenous respiration, \\
interact with membrane \\
phospholipids, inhibit \\
purine uptake, and \\
impair triglyceride or \\
phospholipid \\
biosynthesis (Ishibashi \\
et al., 1990).
\end{tabular} & $\begin{array}{l}\text { Inhibitory but } \\
\text { not cysticidal }\end{array}$ \\
\hline 4 & Fluconazole & & $\begin{array}{l}\text { Inhibits ergosterol } \\
\text { biosynthesis and is } \\
\text { known to inhibit } \\
\text { endogenous respiration, } \\
\text { interact with membrane } \\
\text { phospholipids, inhibit } \\
\text { purine uptake and } \\
\text { impair triglyceride } \\
\text { and/or phospholipid } \\
\text { biosynthesis (Lamb et } \\
\text { al., 2015) }\end{array}$ & \\
\hline 5 & Ketoconazole & & & \begin{tabular}{|l} 
Less effective as \\
compared to \\
fluconazole and \\
itraconazole \\
(Tiewcharoen et \\
al., 2002)
\end{tabular} \\
\hline
\end{tabular}




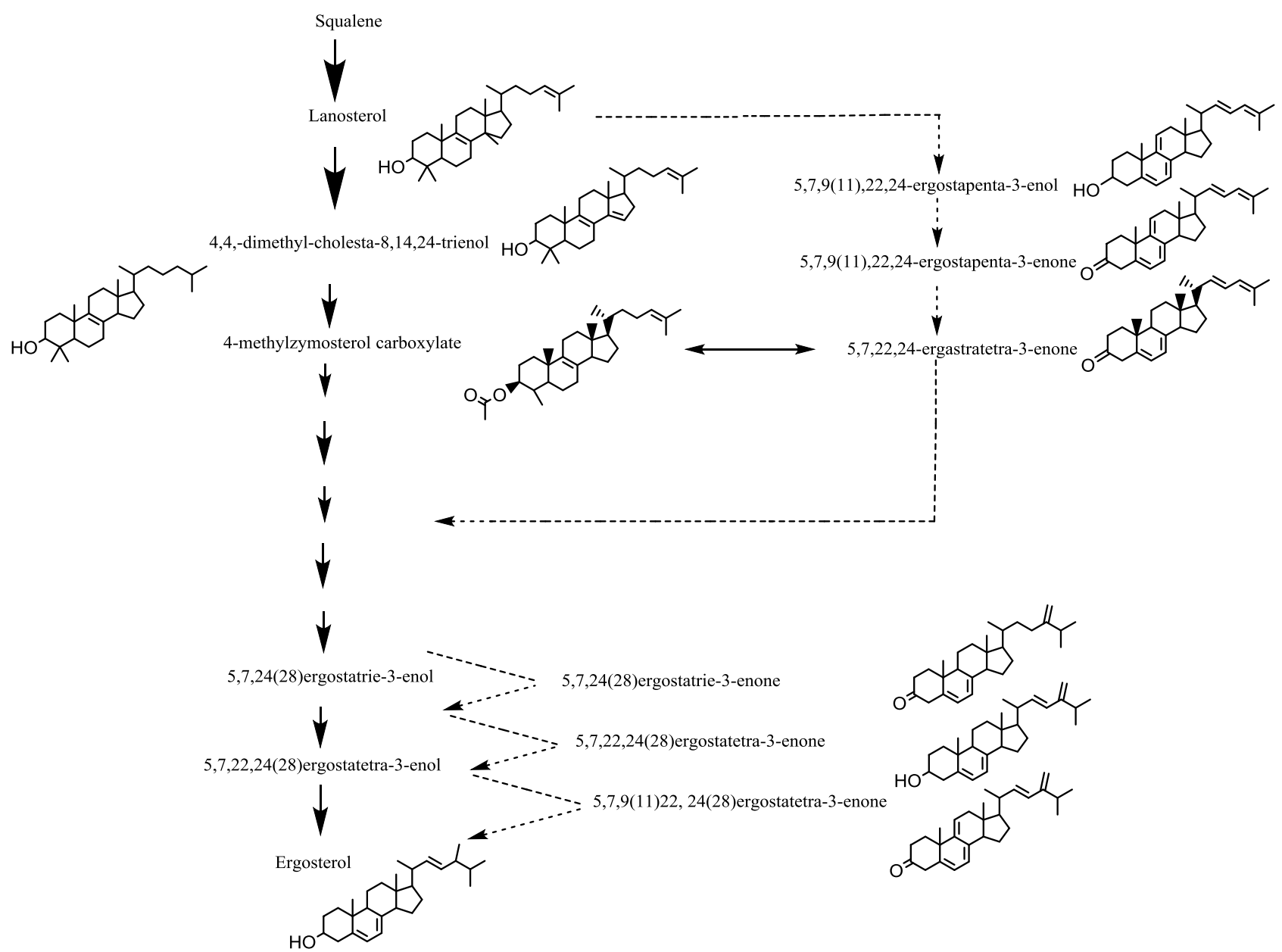

Fig. 1. Scheme of sterol biosynthesis in Acanthamoeba adapted from (Thomson et al., 2017). All these products were identified by GC-MS.

\subsection{Current molecular drug targets in Acanthamoeba}

An interesting approach to the prediction of potential drug targets, designated "differential genome display" has been proposed by Huynen and co-workers (Huynen et al., 1997). The approach relies on the fact that the genomes of parasites generally code for fewer proteins than the genomes of free-living organisms. The genes that are present in the genome of the parasites, but absent in the human host genome, are therefore likely to be considered as candidate drug targets (Chaudhary and Roos, 2005).

Acanthamoeba spp. belongs to eukaryotes, therefore they share functional homologies with the mammalian cells. As many of the drugs cannot be prescribed at effective concentrations due to their unwanted adverse effects. This is particularly relevant for the treatment of amoebal brain infection, where drugs are given intravenously and are expected to cross the blood-brain barrier to access the central nervous system for targeting the intracerebral parasite. In this process, drugs penetrate many other tissues in the body and can affect their physiology before reaching the target site at the desired concentration. Hence, there is a need to develop a targeted therapeutic approach (Siddiqui et al., 2016). The target must be essential for growth and viability, and for critical stages of pathogenesis (Sakharkar et al., 2004). Some of the enzymes like trypanothione reductase and PPi-dependent phosphofructokinase had relatively narrow phylogenetic distribution and could be proposed as potential drug targets against $A$, polyphaga (Ondarza, 2007). Several drugs have been reported which targets the cell membrane, intracellular components, nucleic acid-acting drugs, inhibiting protein synthesis, and enzyme acting agents against
Acanthamoeba. The main drug targets that can be identified in human parasites are discussed as follows:

\subsection{Signaling biomolecules}

The mechanism of encystation and excystation in Acanthamoeba encodes the presence of specific signaling molecules such as proteins composing cyst wall (CSP21) (Hirukawa et al., 1998), cellulose synthesis pathway, and polyphenol oxidase (Anwar et al., 2020). The evidence of protein kinase $\mathrm{C}$ like genes, cell cycle proteins (CDK, CDC2b) (Mengue et al., 2019), apoptotic proteins (caspase 1 \& 3, MCA Atg3, Atg8, etc.) (Kosec et al., 2006; Meslin et al., 2007), signaling pathways such as PI3K, MAP kinase (Siddiqui et al., 2010) provides us with the potential molecular drug targets in Acanthamoeba (Anwar et al., 2020).

\subsection{Enzymes}

Amoebae produce a variety of proteases that can participate in the damage of corneal tissue. Amoebic proteolytic enzymes include serine proteases (Hadas and Mazur, 1993; Mitra et al., 1995), contact-dependent metalloproteases (Khan et al., 2000), elastases (Ferrante and Bates, 1988), cysteine proteases (Wu et al., 2018), and cytotoxic proteases induced by mannose-mediated adhesion (Leher et al., 1998). Proteinases play an important role in various biological actions in Acanthamoeba, including host tissue destruction, pathogenesis, and digestion of phagocytosed food (Hong et al., 2002; Kim et al., 2006; Serrano-Luna et al., 2006). The cysteine and serine proteases are regarded as the major drug targetsMcGrath, 1999). In Acanthamoeba, the 
cysteine protease inhibitor E64d was found to slow encystment and inhibit proteolytic activity (Leitsch et al., 2010).

Other enzymes involved in the metabolic pathways of Acanthamoeba, especially during the transition period of cyst into trophozoite and vice versa, have also been identified (Anwar et al., 2020), which include isocitrate lyase and dehydrogenase (Mehdi and Garg., 1987), glycolate, S-adenosyl- L-methionine decarboxylase (Hugo and Byers., 1993), phospholipase A2 (Mortazavi et al., 2011) fructose bisphosphate aldolase and enolase (Bouyer et al., 2009).

\subsection{Sterols}

De novo sterol biosynthesis from squalene takes place in the most eukaryotes and in lower eukaryotes with an aerobic lifestyle and this reaction occurs in the endoplasmic reticulum (Desmond and Gribaldo, 2009). Sterols also act as a precursor for regulatory molecules that modulate growth, division, differentiation and development processes (Lepesheva and Waterman, 2007; Nes, 2011). Ergosterol is the major end-product of sterol biosynthesis in Acanthamoeba. There was no evidence of cholesterol, desmosterol, campesterol, stigmasterol or 7dehydrostigmasterol. Number of canonical ergosterol precursors was found including lanosterol, 4,4-dimethyl-cholesta-8, 14,24-trienol, 4methyl-zymosterol carboxylate, 5,7,24(28) ergostatetra-3-enol. However 14-dimethyl lanosterol was not detected instead 4,4, dimethyl cholesta-8-ene which is likely reversible intermediate derivative of 14dimethyl lanosterol was detected (Thomson et al., 2017). A summary of the sterol biosynthesis pathway in Acanthamoeba spp. is presented in Figure 1.

\subsection{Azoles as sterol targeting agents against Acanthamoeba}

Since azoles inhibit the synthesis of sterols in fungi by inhibiting cytochrome P450 dependent 14 $\alpha$-lanosterol, the presence of ergosterol in the membrane would account for the sensitivity of Acanthamoeba to the azole compounds. Hence, the isolates of Acanthamoeba spp., were assessed in the presence of five different azoles such as econazole, miconazole, sulconazole, tioconazole and voriconazole. Except for the voriconazole other azoles had no effect or had very little effect on the strains. Voriconazole was the most effective of the drugs tested which could induce actual cell death. Ergosterol levels were reduced in voriconazole tested cultures and inhibited Acanthamoeba 14a-demethylase and resulted in inhibition of ergosterol production (Thomson et al., 2017). Although sterol biosynthesis involves multiple steps, so far only two of them have become major targets for systemic clinical drugs. Statins (cholesterol-lowering agents), which act upstream of the pathway, at the step of mevalonate production (Superko et al., 2012), while azoles, inhibitors of CYP51, serve as the most widely used antifungals (Denning and Bromley, 2015; Lass-Flörl, 2011). Flucoazole (oral) and ketoconazole (systemic) have been used for the treatment of Acanthamoeba keratitis (Amoils and Heney, 1999; Cerva, 1989), whereas clotrimazole can be helpful in controlling recurrent infections after penetrating keratoplasty (Driebe et al., 1988). A recent review published by Elsheikha et al., 2020 also discussed that the use of oral voricanzole in combination therapy with miltefosine, has shown to decrease the size of brain lesions and serological titres in an immunocompetent patient having GAE a study done by (Webster et al., 2012). Although, many review articles have been published on the treatment of Acanthamoeba infections, but this is the first review of its kind which focuses only on the azoles and their synthetic libraries along with their nanoconjugation for their potential use against Acanthamoeba.

\subsection{Use of nanoparticles to improve drug efficacy}

Nanoparticles are the elementary structures of nanotechnology and are important materials for fundamental studies and various applications including their bioactivities (Patil et al., 2012). Synthesis of a variety of drug particle of nano-size along with their specific physical and chemical properties has been involved in the preparation of novel therapeutics (Brigger et al., 2012; Merisko-Liversidge et al., 2003). By controlling the definite structure of nanoscale dimensions, their surface structure can be modified, which can help in the improved bioavailability of poorly absorbed drugs and a drug can be delivered efficiently.

The development of novel and efficient nanoparticle-based antimicrobial drugs against resistant microbes is among the major interests in biomedical research (Rai et al., 2012). Nanoparticles used as drug delivery agents are generally $<100 \mathrm{~nm}$ in dimension and consist of different biodegradable materials such as natural or synthetic polymers, lipids, or metals. Nanoparticles are taken up by cells more efficiently than micro molecules and therefore could be used as effective transport and delivery systems (Suri et al., 2007). By loading drugs into nanoparticles through physical encapsulation, adsorption, or chemical conjugation, the pharmacokinetics and therapeutic index of the drugs can be significantly improved (Zhang et al., 2010). A few types of nanoparticles including liposomes, polymeric nanoparticles, solid lipid nanoparticles and dendrimers have been widely investigated as antimicrobial drug delivery platforms. The most widely used nanoparticles include gold, silver, titanium oxide and iron nanoparticles (ElAnsary and Al-Daihan, 2009). Figure 2 summarizes the advantages of nanoparticles in suitable drug delivery applications.

\subsection{Nanoparticles used against Acanthamoeba}

Gold nanoparticles are well suited for a wide range of biological applications because of their unique range of biological applications because of their physical and chemical properties (Pissuwan et al., 2010). As gold is inert, it exhibits weak cytotoxic effects; thus it is considered as the nanoparticle of choice when performing conjugations with various biomolecules and ligands to develop strategies for targeting pathogens (Connor et al., 2005). At present, there are only few reports for the use of nanoparticles against free living amoeba. Among metal nanoparticles, gold and silver conjugated with different drugs and natural compounds have been effective against $A$. castellanii (Anwar et al., 2018c; Aqeel et al., 2016; Padzik et al., 2018., Niyyati et al., 2018). In a recent study, gold conjugated nanoparticle enhanced the effect of chlorhexidine gluconate against antiacanthamoebic drugs. Amoebicidal assays performed revealed that although gold conjugated chlorhexidine and chlorhexidine alone exhibited amoebicidal properties but gold conjugated chlorhexidine

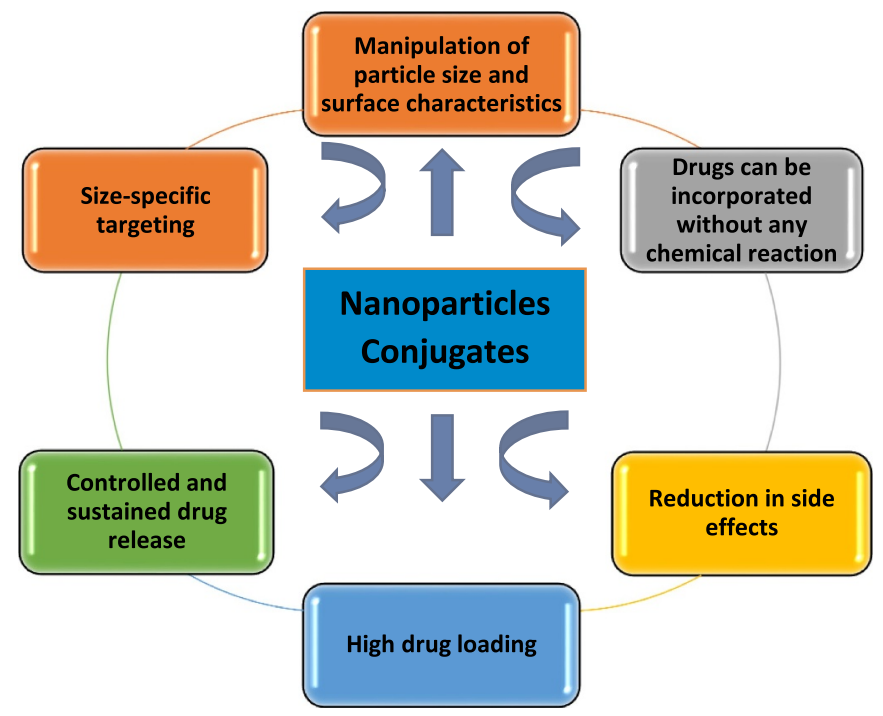

Fig. 2. Effects of nanoparticle on drug delivery. 


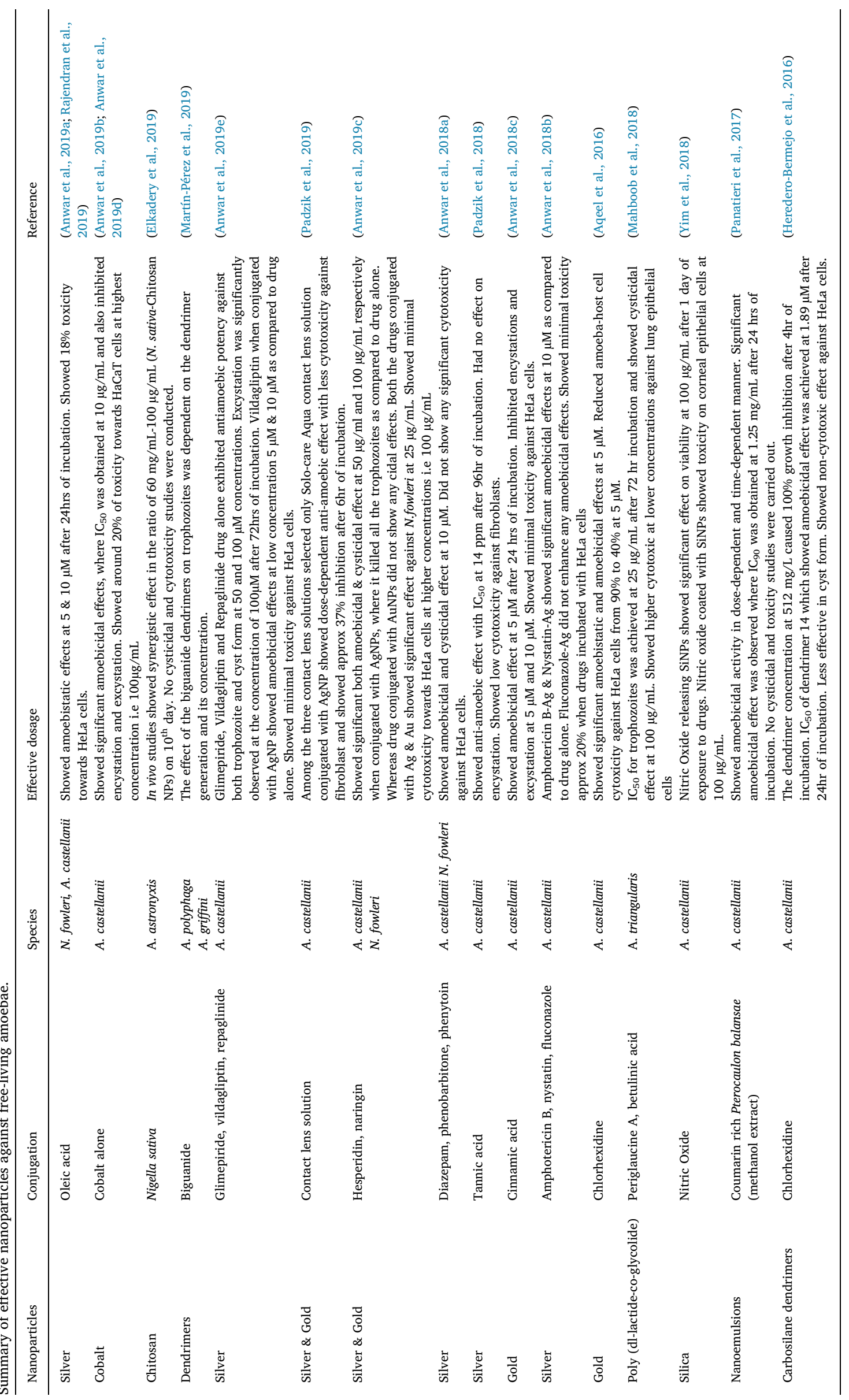


showed higher toxicity against $A$. castellani than chlorhexidine alone (Aqeel et al., 2016). The increased cytotoxicity of gold nanoparticles is due to the high reactivity of nanoparticles with living cells as well as easy translocation of drugs into the living cells enhancing drug efficacy (Dykman and Khlebtsov, 2011). In recent studies by our research team, it was reported that the enhanced effects of antifungal drugs nystatin, fluconazole, amphotericin B conjugated with gold nanoparticles on $A$. castellanii. Since these drugs target ergosterol pathway which is an essential component of $A$. castellanii membrane, their conjugation with nanoparticles resulted in the increased bioactivity (Anwar et al., 2019f).

In another research by our team reported that the cinnamic acid (CA) conjugated with gold showed the enhanced effect of anti-acanthmoebic activity (Anwar et al., 2018c). As CA is a natural organic compound that is found in variety of plants and chemical constituent of cinnamon and has antimicrobial and antibacterial properties.

Cobalt is another metal which has shown potential as for several biological studies (Czarnek et al., 2015). In one of the studies done by our team, we have shown antiacanthamoebic effects of different cobalt nanoparticles against trophozoites and cysts. Three different compositions of $\mathrm{CoNPs}\left(\mathrm{Co}_{3} \mathrm{O}_{4}, \mathrm{Co}(\mathrm{OH})_{2}, \mathrm{Co}_{3}\left(\mathrm{PO}_{4}\right)_{2}\right)$ were tested against $A$. castellanii. The smallest sized granular cobalt oxide NPs showed minimum anti-amoebic effects as compared to $\mathrm{Co}_{3}\left(\mathrm{PO}_{4}\right)_{2}$, and $\mathrm{Co}(\mathrm{OH})_{2}$ which showed better overall effects (Anwar et al., 2019d).

The plasma membrane of Acanthamoeba is made up of $25 \%$ phospholipids (Siddiqui and Khan, 2012) and oleic acid was found to be amongst the most abundant fatty acid in every phospholipid class (Palusinska-Szysz et al., 2014). Thus, fatty acids have also been known to possess antimicrobial and antibacterial properties (Desbois and Smith, 2010). A research done on oleic acid against Acanthamoeba have shown significant antiacanthamoebic effects, but oleic acid conjugated with silver nanoparticles have exhibited better effects than oleic acid alone. Moreover, oleic acid has shown only $18 \%$ toxicity to HeLa cells and this makes OA and OA-AgNPs safer alternatives against Acanthamoeba infections (Anwar et al., 2019a).

In another report, antidiabetic drugs like Glimepiride, Vildagliptin and Repaglinide were tested against $A$. castellanii and they were conjugated with AgNPs to enhance their antiacanthamoebic activity. All three drugs showed significant anti-amoebic effects and blocked encystation. Vildagliptin-AgNPs have exhibited antiacanthamoebic effects on both trophozoites and cysts form at much-reduced concentrations. Hence these antidiabetic drugs may serve as a potential drug target in the treatment and management of $A$. castellanii infections (Anwar et al., 2019e).

Acanthamoeba keratitis incidents are mostly happening among the contact lens wearers and as the number of cases is increasing and often applied therapy is unsuccessful, proper hygiene and effective contact lenses disinfection are crucial for the prevention of the disease. There is a need to enhance the disinfecting activity of contact lens solutions to prevent amoebic infections. Studies done by (Padzik et al., 2019) and his team have observed in their research that anti-amoebic activity was enhanced when the contact lens solutions were conjugated with gold and silver nanoparticles with low cytotoxicity.

Some plant metabolites such as flavonoids, alkaloids or terpenes present anti-parasitic activity and among them, tannins are polyphenolic plant metabolites with confirmed anti-obesity, anti-diabetes, antioxidant and anti-microbial activities (El-Sayed et al., 2012; Hajaji et al., 2017). In one of the studies tannic acid-modified silver nanoparticles (AgTANPs), pure silver nanoparticles (AgNPs) and pure gold nanoparticles (AuNPs) was investigated against strains of Acanthamoeba spp. AgTANPs were well absorbed by the trophozoites and did not induce encystation. The most significant anti-amoebic effect in relation to cytotoxicity was observed in AgTANPs against Neff and P13 clinical strain for which $\mathrm{IC}_{50}$ was the most significant in relation to cytotoxicity. Hence AgTANPs were more significant than pure AgNPs and AuNPs (Padzik et al., 2018). In recent reports, titanium oxide nanoparticles have shown in vitro antiacanthamoebic effects triggered by ultraviolet radiations (Gomart et al., 2018), while doping with zinc oxide nanoparticles have shown improved photochemotherapy (Imran et al., 2016).

Effects of AgNPs conjugated with amphotericin B, nystatin, and fluconazole against $A$. castellanii has also been studied by our team. Amoebicidal results in this research revealed that drug-coated AgNPs are more effective as compared to drug alone. Nys-AgNPs showed remarkable anti-amoebic effects at both 10 and $5 \mu \mathrm{M}$ concentrations. Drug coated with AgNPs pre-treated with amoeba resulted in significant decrease in host cell cytotoxicity (Anwar et al., 2018b). The effects of different nanoparticles which have so far been found effective against Acanthamoeba and other free-living amoebae are summarized in Table 2. Nanoparticles conjugates have shown promising results against free-living amoebae, however, none of the studies so far have identified their in vivo potential or exact mode of action. These gaps in the research are currently the limiting factors in the development of nanomedicine against infections caused by free-living amoebae.

\subsection{Perspective}

Although recent studies have progressed the understanding of the biology of FLA protists and their detection in human hosts, but our knowledge of virulence factor and mechanisms of pathogenesis remains unclear. For the past several decades, there has been little improvement in the morbidity and mortality associated with Acanthamoeba diseases. The high mortality is due to the lack of familiarity with these amoebic diseases, delay in diagnosis and lack of optimal antimicrobial therapy. Correct diagnosis and treatment of these diseases has been a complicated issue for the researchers all over the world. Hence, there is an urgent need for an improved understanding of the possibilities for therapeutic actions towards the pathophysiology and pathogenesis of Acanthamoeba. Novel targeted drug therapy remains the only viable option to tackle these diseases. On the other hand, nanoparticles play an important role in drug delivery platforms where they enhance the efficacy of the drugs. However, at present there are only few reports for the use of nanoparticles against free living amoeba. This review summarizes the importance and current progress of the azoles and nanoparticles, as a major potential combination for the advancement in the antimicrobial chemotherapy against the diseases caused by Acanthamoeba.

One of the pivotal drugs target against Acanthamoeba is sterol biosynthesis pathway, where azoles are known to inhibit the action of cytochrome P450 dependent $14 \alpha$ lanosterol which leads to cell lysis/ cell necrosis. Hence, azole compounds play an important role in biosynthesis pathways and thus they can bind easily with the enzymes and receptors in organisms through weak interactions thereby exhibiting anti-amoebic properties. Heterocyclic scaffolds present in natural as well as synthetic compounds possess a diverse range of biological activities. They play an important role in biochemical processes because the side groups of the most typical and essential constituents of living cells, DNA and RNA, are based on aromatic heterocycles (Dua et al., 2011). Azoles are subdivided into several classes including pyrazoles, imidazoles, triazoles, tetrazoles, oxazole, thiazole etc., however, most of the azole groups operate via a common mode of action, they prevent the synthesis of ergosterol, which is the major component of plasma membranes in fungi and FLA. Several azole compounds like voriconazole, fluconazole, ketoconazole, itraconazole etc., have been tested against trophic stages of Acanthamoeba isolates which were found to have potent inhibitory effects on amoebae growth and in some cases their viability. Amongst different azoles, some case studies have suggested that the voriconazole can be considered as a strong agent for the treatment of the $\mathrm{AK}$, as it inhibits the proliferation of trophozoites and benefits from being easily administered either orally as tablets or topically in eye drops (Tu et al., 2010). However, besides some commonly known azole antifungal drugs, no work has been done to optimize the lead compounds on synthetic libraries of azoles by utilizing 
medicinal chemistry approach. Screening of libraries of synthetic azoles may prove to be an effective targeted therapeutic approach against Acantheamoeba infections, as described above by the reported evidence.

Hence, based upon the discussed information in this review, we suggest that azole scaffolds can be modified to create a new range of azole therapeutics which may lead to increased effectiveness of the drug potency against Acanthamoeba. With the above suggested investigations, the azoles derivatives may act as a new leading drug against Acanthamoebic infections. In our continued studies, we have been trying to optimise the diverse range of azole scaffolds with and without the conjugation with nanoparticles for the drug development and delivery. We expect that this combinatorial approach of using diversified azoles scaffolds with the conjugated nanoparticles may lead to the development of novel therapeutic agents against this pathogenic parasite Acanthamoeba castellanii.

\section{Funding}

Not applicable.

\section{Ethical approval}

Not applicable.

\section{Conflict of interest}

None.

\section{Acknowledgments}

This work was supported by Sunway University.

\section{References}

Adagu, I.S., Nolder, D., Warhurst, D.C., Rossignol, J.-F., 2002. In vitro activity of nitazoxanide and related compounds against isolates of Giardia intestinalis, Entamoeba histolytica and Trichomonas vaginalis. J. Antimicrob. Chemother. 49, 103-111.

Amoils, S.P., Heney, C., 1999. Acanthamoeba keratitis with live isolates treated with cryosurgery and fluconazole.Am. J. Opthalmol. 127, 718-720.

Anderluh, P.Š., Vilfan, G., Preželj, A., Urleb, U., 2009. Synthesis of C6-ethylidene meropenem derivative with antimicrobial activity. Acta Chim. Sloven 56.

Anwar, A., Siddiqui, R., Khan, N.A., 2020. Whole Organism model to study molecular mechanisms of differentiation and dedifferentiation. Biology 9 (4), 79.

Anwar, A., Abdalla, S.A.O., Aslam, Z., Shah, M.R., Siddiqui, R., Khan, N.A., 2019a. Oleic acid-conjugated silver nanoparticles as efficient anti-amoebic agent against Acanthamoeba castellanii. Parasitol. Res. 1-10.

Anwar, A., Chi Fung, L., Anwar, A., Jagadish, P., Numan, A., Khalid, M., Shahabuddin, S., Siddiqui, R., Khan, N.A., 2019b. Effects of shape and size of cobalt phosphate nanoparticles against Acanthamoeba castellanii. Pathogens 8, 260.

Anwar, A., Masri, A., Rao, K., Rajendran, K., Khan, N.A., Shah, M.R., Siddiqui, R., 2019c. Antimicrobial activities of green synthesized gums-stabilized nanoparticles loaded with flavonoids. Sci. Rep. 9, 3122

Anwar, A., Numan, A., Siddiqui, R., Khalid, M., Khan, N.A., 2019d. Cobalt nanoparticles as novel nanotherapeutics against Acanthamoeba castellanii. Parasit. Vectors 12, 280.

Anwar, A., Rajendran, K., Siddiqui, R., Raza Shah, M., Khan, N.A., 2018a. Clinically approved drugs against CNS diseases as potential therapeutic agents to target braineating amoebae. ACS Chem. Neurosci. 10, 658-666.

Anwar, A., Siddiqui, R., Hussain, M.A., Ahmed, D., Shah, M.R., Khan, N.A., 2018b. Silver nanoparticle conjugation affects antiacanthamoebic activities of amphotericin B, nystatin, and fluconazole. Parasitol. Res. 117, 265-271.

Anwar, A., Siddiqui, R., Shah, M.R., Khan, N.A., 2018c. Gold nanoparticle-conjugated cinnamic acid exhibits antiacanthamoebic and antibacterial properties. Antimicrob. Agents Chemother. 62 e00630-e00618.

Anwar, A., Siddiqui, R., Shah, M.R., Khan, N.A., 2019e. Antidiabetic drugs and their nanoconjugates repurposed as novel antimicrobial agents against Acanthamoeba castellanii. J. Microbiol. Biotechnol 29, 713-720.

Anwar, A., Siddiqui, R., Shah, M.R., Khan, N.A., 2019f. Gold nanoparticles conjugation enhances antiacanthamoebic properties of nystatin, fluconazole and amphotericin B. J. Microbiol. Biotechnol. 29, 171-177.

Aqeel, Y., Siddiqui, R., Anwar, A., Shah, M.R., Khan, N.A., 2016. Gold nanoparticle conjugation enhances the antiacanthamoebic effects of chlorhexidine. Antimicrob. Agents Chemother. 60, 1283-1288.

Ashok, M., Holla, B.S., Poojary, B., 2007. Convenient one pot synthesis and antimicrobial evaluation of some new Mannich bases carrying 4-methylthiobenzyl moiety. Eur. J. Med. Chem. 42, 1095-1101.
Azam, A., Agarwal, S., 2007. Targeting Amoebiasis: status and developments. Curr. Bioact. Comp. 3, 121-133.

Berger, S.T., Mondino, B.J., Hoft, R.H., Donzis, P.B., Holland, G.N., Farley, M.K., Levenson, J.E., 1990. Successful medical management of Acanthamoeba keratitis. Am. J. Ophthalmol 110, 395-403.

Bhat, A.R., Athar, F., Azam, A., 2009. New derivatives of 3, 5-substituted-1, 4, 2-dioxazoles: synthesis and activity against Entamoeba histolytica. Eur. J. Med. Chem. 44, 926-936.

Bodey, G.P., 1992. Azole antifungal agents. Clin. Infect. Dis 14, S161-S169.

Bouyer, S., Rodier, M.H., Guillot, A., Héchard, Y., 2009. Acanthamoeba castellanii: Proteins involved in actin dynamics, glycolysis, and proteolysis are regulated during encystation. Exp. Parasitol. 123, 90-94.

Brigger, I., Dubernet, C., Couvreur, P., 2012. Nanoparticles in cancer therapy and diagnosis. Adv. Drug Deliver. Rev. 64, 24-36.

Bryskier, A., 2005. Antimicrobial agents: antibacterials and antifungals. ASM press.

Casper, T., Basset, D., Leclercq, C., Fabre, J., Peyron-Raison, N., Reynes, J., 1999. Disseminated Acanthamoeba infection in a patient with AIDS: response to 5-fluorocytosine therapy. Clin. Infect. Dis 29, 944-945.

Cerva, L., 1989. Acanthamoeba culbertsoni and Naegleria fowleri: occurrence of antibodies in man. J. Hyg. Epidemiol. Microbiol. Immunol. 33, 99-103.

Chaudhary, K., Roos, D.S., 2005. Protozoan genomics for drug discovery. Nat. Biotechnol 23, 1089.

Chavez, D.E., Parrish, D.A., 2009. New heterocycles from tetrazines and oxadiazoles. J. Heterocycl. Chem. 46, 88-90.

Çolak, A.T., Çolak, F., Atar, N., Olgun, A., 2010. Synthesis, spectral, thermal analysis, biological activity and kinetic studies of copper (II)-Pyridine-2, 5-dicarboxylate complexes with 2-aminomethylpyridine and 8-hydroxyquinoline. Acta Chim. Sloven. 57.

Connor, E.E., Mwamuka, J., Gole, A., Murphy, C.J., Wyatt, M.D., 2005. Gold nanoparticles are taken up by human cells but do not cause acute cytotoxicity. Small 1, 325-327.

Craigo, W.A., LeSueur, B.W., Skibo, E.B., 1999. Design of highly active analogues of the pyrrolo [1, 2-a] benzimidazole antitumor agents. J. Med. Chem. 42, 3324-3333.

Culbertson, C.G., of, W.t.T.A., Overton, W.M., Reveal, M.A., 1961. Pathogenic Acanthamoeba (Hartmanella). Am. J. Clin. Pathol. 35, 195-202.

Czarnek, K., Terpiłowska, S., Siwicki, A.K., 2015. Selected aspects of the action of cobalt ions in the human body. Cent-Eur. J. Immunol. 40, 236.

D'Aversa, G., Stern, G.A., Driebe, W.T., 1995. Diagnosis and successful medical treatment of Acanthamoeba keratitis. Arch. Ophthalmol 113, 1120-1123.

De Jonckheere, J.F., 1991. Ecology of Acanthamoeba. Rev. Infect. Dis 13, S385-S387.

Deluoi, A.M., Teilhac, M.F., Poirot, J.L., Maslo, C., Luboinski, J., Rozenbaum, W., Chatelet, F.P., 1996. Cutaneous lesions due to Acanthamoeba sp in a patient with AIDS. J. Eukaryot. Microbiol. 43, 130S-131S.

Denning, D.W., Bromley, M.J., 2015. How to bolster the antifungal pipeline. Science 347 1414-1416.

Desbois, A.P., Smith, V.J., 2010. Antibacterial free fatty acids: activities, mechanisms of action and biotechnological potential. Appl. Microbiol. Biotechnol. 85, 1629-1642.

Desmond, E., Gribaldo, S., 2009. Phylogenomics of sterol synthesis: insights into the origin, evolution, and diversity of a key eukaryotic feature. Gen. Biol. Evol. 1 , 364-381.

Driebe, J.T.W., Stern, G.A., Epstein, R.J., Visvesvara, G.S., Adi, M., Komadina, T., 1988 Acanthamoeba keratitis. Potential role for topical clotrimazole in combination chemotherapy. Arch. Ophthalmol. 106, 1196-1201.

Dua, R., Shrivastava, S., Sonwane, S., Srivastava, S., 2011. Pharmacological significance of synthetic heterocycles scaffold: a review. Adv. Biol. Res. 5, 120-144.

Dudley, R., Jarroll, E.L., Khan, N.A., 2009. Carbohydrate analysis of Acanthamoeba castellanii. Exp. Parasitol 122, 338-343.

Duma, R.J., Finley, R., 1976. In vitro susceptibility of pathogenic Naegleria and Acanthamoeba species to a variety of therapeutic agents. Antimicrob. Agents Chemother. 10, 370-376.

Dykman, L., Khlebtsov, N., 2011. Gold nanoparticles in biology and medicine: recent advances and prospects. Acta Natur. (англоязычная версия) 3.

El-Ansary, A., Al-Daihan, S., 2009. On the toxicity of therapeutically used nanoparticles: an overview. J. Toxicol. 2009.

El-Sayed, N.M., Ismail, K.A., Ahmed, S.A.-E.-G., Hetta, M.H., 2012. In vitro amoebicidal activity of ethanol extracts of Arachis hypogaea L., Curcuma longa L. and Pancratium maritimum L. on Acanthamoeba castellanii cysts. Parasitol. Res. 110, 1985-1992.

Elkadery, A.A.S., Elsherif, E.A., Eldin, H.M.E., Fahmy, I.A.F., Mohammad, O.S., 2019 Efficient therapeutic effect of Nigella sativa aqueous extract and chitosan nanoparticles against experimentally induced Acanthamoeba keratitis. Parasitol. Res. 1-12.

Elsheikha, H.M., Siddiqui, R., Khan, N.A., 2020. Drug discovery against Acanthamoeba infections: present knowledge and unmet needs. Pathogens 9 (5), 405.

Ferrante, A., Bates, E.J., 1988. Elastase in the pathogenic free-living amoebae Naegleria and Acanthamoeba Spp. Infect. Immun 56, 3320-3321.

Garg, P., Kalra, P., Joseph, J., 2017. Non-contact lens related Acanthamoeba keratitis. Ind. J. Ophthalmol 65, 1079.

Goad, L.J., Berens, R.L., Marr, J.J., Beach, D.H., Holz Jr, G.G., 1989. The activity of ketoconazole and other azoles against Trypanosoma cruzi: biochemistry and chemotherapeutic action in vitro. Mol. Biochem. Parasitol 32, 179-189.

Gomart, G., Denis, J., Bourcier, T., Dory, A., Abou-Bacar, A., Candolfi, E., Sauer, A., 2018. In vitro amoebicidal activity of Titanium dioxide/UV-A combination against Acanthamoeba. Invest. Ophthalmol. Vis. Sci. 59, 4567-4571.

Hadas, E., Mazur, T., 1993. Proteolytic enzymes of pathogenic and non-pathogenic strains of Acanthamoeba spp. Trop. Med. Parasitol.: official organ of Deutsche Tropenmedizinische Gesellschaft and of Deutsche Gesellschaft fur Technische Zusammenarbeit (GTZ)44, 197-200. 
Hajaji, S., Sifaoui, I., López-Arencibia, A., Reyes-Batlle, M., Valladares, B., Pinero, J.E., Lorenzo-Morales, J., Akkari, H., 2017. Amoebicidal activity of $\alpha$-bisabolol, the main sesquiterpene in chamomile (Matricaria recutita L.) essential oil against the trophozoite stage of Acanthamoeba castellanii Neff. Acta Parasitol. 62, 290-295.

Helton, J., Loveless, M., White, J.C., 1993. Cutaneous Acanthamoeba infection associated with leukocytoclastic vasculitis in an AIDS patient. Am. J. Dermatopathol. 15, 146-149.

Heredero-Bermejo, I., Sanchez-Nieves, J., Soliveri, J., Gomez, R., De la Mata, F., CopaPatino, J., Perez-Serrano, J., 2016. In vitro anti-Acanthamoeba synergistic effect of chlorhexidine and cationic carbosilane dendrimers against both trophozoite and cyst forms. Int. J. Pharmaceut. 509, 1-7.

Hirukawa, Y., Nakato, H., Izumi, S., Tsuruhara, T., Tomino, S., 1998. Structure and expression of a cyst specific protein of Acanthamoeba castellanii. Biochim. Biophys. Acta 1398, 47-56.

Hong, Y.-C., Hwang, M.-Y., Yun, H.-C., Yu, H.-S., Kong, H.-H., Yong, T.-S., Chung, D.-I., 2002. Isolation and characterization of a cDNA encoding a mammalian cathepsin Llike cysteine proteinase from Acanthamoeba healyi. Korean J. Parasitol 40, 17.

Hugo, E.R., Byers, T.J., 1993. S-adenosyl-L-methionine decarboxylase of Acanthamoeba castellanii (Neff): purification and properties. Biochem. J. 295, 203-209.

Huynen, M.A., Diaz-Lazcoz, Y., Bork, P., 1997. Differential genome display. Trends Gen 10, 389-390.

Imran, M., Muazzam, A.G., Habib, A., Matin, A., 2016. Synthesis, characterization and amoebicidal potential of locally synthesized $\mathrm{TiO} 2$ nanoparticles against pathogenic Acanthamoeba trophozoites in vitro. J. Photochem. Photobiol. B: Biol. 159, 125-132.

Ishibashi, Y., Matsumoto, Y., Kabata, T., Watanabe, R., Hommura, S., Yasuraoka, K., Ishii, K., 1990. Oral itraconazole and topical miconazole with debridement for Acanthamoeba keratitis. Am. J. Ophthalmol 109, 121-126.

Jones, D., Visvesvara, G., Robinson, N., 1975. Acanthamoeba polyphaga keratitis and Acanthamoeba uveitis associated with fatal meningoencephalitis. Trans. Ophthalmol. Soc. UK. 95, 221-232.

Khan, N.A., 2006. Acanthamoeba: biology and increasing importance in human health FEMS Microbiol. Rev. 30, 564-595.

Khan, N.A., Anwar, A., Siddiqui, R., 2017. Future priorities in tackling infections due to brain-eating amoebae. ACS Chem. Neurosci. 8 2355-2355.

Khan, N.A., Jarroll, E.L., Panjwani, N., Cao, Z., Paget, T.A., 2000. Proteases as markers for differentiation of pathogenic and nonpathogenic species of Acanthamoeba. J. Clin. Microbiol. 38, 2858-2861.

Kim, W.-T., Kong, H.-H., Ha, Y.-R., Hong, Y.-C., Jeong, H.J., Yu, H.S., Chung, D.-I., 2006. Comparison of specific activity and cytopathic effects of purified $33 \mathrm{kDa}$ serine proteinase from Acanthamoeba strains with different degree of virulence. Korean. J. Parasitol. 44, 321

Kosec, G., Alvarez, V.E., Agüero, F., Sánchez, D., Dolinar, M., Turk, B., Turk, V., Cazzulo, J.J., 2006. Metacaspases of trypanosoma cruzi: possible candidates for programmed cell death mediators. Mol. Biochem. Parasitol. 145, 18-28.

Kulsoom, H., Baig, A.M., Siddiqui, R., Khan, N.A., 2014. Combined drug therapy in the management of granulomatous amoebic encephalitis due to Acanthamoeba spp., and Balamuthia mandrillaris. Exp. Parasitol 145, S115-S120.

Kumar, R., Lloyd, D., 2002. Recent advances in the treatment of Acanthamoeba keratitis. Clin. Infect. Dis 35, 434-441.

Lamb, D.C., Warrilow, A.G., Rolley, N.J., Parker, J.E., Nes, W.D., Smith, S.N., Kelly, D.E., Kelly, S.L., 2015. Azole antifungal agents to treat the human pathogens Acanthamoeba castellanii and Acanthamoeba polyphaga through inhibition of sterol $14 \alpha$-demethylase (CYP51). Antimicrob. Agents Chemother 59, 4707-4713.

Lass-Flörl, C., 2011. Triazole antifungal agents in invasive fungal infections. Drugs 71, 2405-2419.

Leher, H., Silvany, R., Alizadeh, H., Huang, J., Niederkorn, J.Y., 1998. Mannose induces the release of cytopathic factors from Acanthamoeba castellanii. Infect. Immun. 66, 5-10.

Leitsch, D., Köhsler, M., Marchetti-Deschmann, M., Deutsch, A., Allmaier, G., Duchêne, M., Walochnik, J., 2010. Major role for cysteine proteases during the early phase of Acanthamoeba castellanii encystment. Eukaryot. Cell 9, 611-618.

Lepesheva, G.I., Waterman, M.R., 2007. Sterol 14 $\alpha$-demethylase cytochrome P450 (CYP51), a P450 in all biological kingdoms. Biochim. Biophys. Acta (BBA)-Gen. Subjects 1770, 467-477.

Lorenzo-Morales, J., Khan, N.A., Walochnik, J., 2015. An update on Acanthamoeba keratitis: diagnosis, pathogenesis and treatment. Parasite 22.

Mahboob, T., Nawaz, M., Tian-Chye, T., Samudi, C., Wiart, C., Nissapatorn, V., 2018 Preparation of poly (dl-lactide-co-glycolide) nanoparticles encapsulated with periglaucine A and betulinic acid for in vitro anti-Acanthamoeba and cytotoxicity activities. Pathogens 7, 62

Marciano-Cabral, F., Cabral, G., 2003. Acanthamoeba spp. as agents of disease in humans. Clin. Microbiol. Rev. 16, 273-307.

Martín-Pérez, T., Lozano-Cruz, T., Criado-Fornelio, A., Ortega, P., Gómez, R., de la Mata, F., Pérez-Serrano, J., 2019. Synthesis and in vitro activity of new biguanide-containing dendrimers on pathogenic isolates of Acanthamoeba polyphaga and Acanthamoeba griffini. Parasitol. Res 118, 1953-1961.

McGrath, M.E., 1999. The lysosomal cysteine proteases. Ann. Rev. Biophys. Biomol. Struct. 28,181

Mehdi, H., Garg, N.K., 1987. Changes in the lipid composition and activities of isocitrate dehydrogenase and isocitrate lyase during encystation of Acanthamoeba culbertsoni strain A-1. Trans. Royal Soc. Trop. Med. Hyg. 81, 633-636.

Mengue, L., Régnacq, M., Aucher, W., Portier, E., Héchard, Y., Samba-Louaka, A., 2019. Legionella pneumophila prevents proliferation of its natural host Acanthamoeba castellanii. Sci. Rep 6, 1-12.

Merisko-Liversidge, E., Liversidge, G.G., Cooper, E.R., 2003. Nanosizing: a formulation approach for poorly-water-soluble compounds. Eur. J. Pharm. Sci. 18, 113-120.
Meslin, B., Barnadas, C., Boni, V., Latour, C., De Monbrison, F., Kaiser, K., Picot, S., 2007. Features of apoptosis in Plasmodium falciparum erythrocytic stage through a putative role of PfMCA1 metacaspase-like protein. J. Inf. Dis. 195, 1852-1859.

Mitra, M.M., Alizadeh, H., Gerard, R.D., Niederkorn, J.Y., 1995. Characterization of a plasminogen activator produced by Acanthamoeba castellanii. Mol. Biochem. Parasitol 73, 157-164.

Mortazavi, P.N., Keisary, E., Loh, L.N., Jung, S.Y., Khan, N.A., 2011. Possible roles of phospholipase A2 in the biological activities of Acanthamoeba castellanii (T4 genotype). Protist 162, 168-176.

Mukherjee, T., Boshoff, H., 2011. Nitroimidazoles for the treatment of TB: past, present and future. Fut. Med. Chem. 3, 1427-1454.

Nagle, A.S., Khare, S., Kumar, A.B., Supek, F., Buchynskyy, A., Mathison, C.J., Chennamaneni, N.K., Pendem, N., Buckner, F.S., Gelb, M.H., 2014. Recent developments in drug discovery for leishmaniasis and human African trypanosomiasis. Chem. Rev. 114, 11305-11347.

Nes, W.D., 2011. Biosynthesis of cholesterol and other sterols. Chem. Rev. 111, 6423-6451.

Niederkorn, J.Y., 2002. The role of the innate and adaptive immune responses in Acanthamoeba keratitis. Arch. Immunol. Ther. Exper-Eng. Ed. 50, 53-60.

Niyyati, M., Sasani, R., Mohebali, M., GHAZIKHANSARI, M., Kargar, F., Hajialilo, E., Rezaeian, M., 2018. Anti-Acanthamoeba effects of silver and gold nanoparticles and contact lenses disinfection solutions. Iran. J. Parasitol. 13 (2), 180.

Ondarza, R., 2007. Drug targets from human pathogenic amoebas: Entamoeba histolytica, Acanthamoeba polyphaga and Naegleria fowleri. Infect. Disord.-Drug Targets (Formerly Curr. Drug Targets-Infect. Disord.)7, 266-280.

Orozco, L., Hanigan, W., Khan, M., Fratkin, J., Lee, M., 2011. Neurosurgical intervention in the diagnosis and treatment of Balamuthia mandrillaris encephalitis: report of 3 cases. J. Neurosurg. 115, 636-640.

Padzik, M., Hendiger, E.B., Chomicz, L., Grodzik, M., Szmidt, M., Grobelny, J., LorenzoMorales, J., 2018. Tannic acid-modified silver nanoparticles as a novel therapeutic agent against Acanthamoeba. Parasitol. Res 117, 3519-3525.

Padzik, M., Hendiger, E.B., Żochowska, A., Szczepaniak, J., Baltaza, W., PietruczukPadzik, A., Olędzka, G., Chomicz, L., 2019. Evaluation of in vitro effect of selected contact lens solutions conjugated with nanoparticles in terms of preventive approach to public health risk generated by Acanthamoeba strains. Ann. Agric. Environ. Med.: AAEM 26, 198-202.

Palusinska-Szysz, M., Kania, M., Turska-Szewczuk, A., Danikiewicz, W., Russa, R., Fuchs, B., 2014. Identification of unusual phospholipid fatty Acyl compositions of Acanthamoeba castellanii. PloS One 9, e101243.

Panatieri, L.F., Brazil, N.T., Faber, K., Medeiros-Neves, B., von Poser, G.L., Rott, M.B., Zorzi, G.K., Teixeira, H.F., 2017. Nanoemulsions containing a coumarin-rich extract from Pterocaulon balansae (Asteraceae) for the treatment of ocular Acanthamoebc keratitis. AAPS Pharm. Sci. Tech. 18, 721-728.

Patil, S.V., Borase, H.P., Patil, C.D., Salunke, B.K., 2012. Biosynthesis of silver nanoparticles using latex from few euphorbian plants and their antimicrobial potential. Appl. Biochem. Biotechnol. 167, 776-790.

Peng, X.-M., Cai, G.-X., Zhou, C.-H., 2013. Recent developments in azole compounds as antibacterial and antifungal agents. Curr. Topics Med. Chem. 13, 1963-2010.

Pérez-Santonja, J.J., Kilvington, S., Hughes, R., Tufail, A., Matheson, M., Dart, J.K., 2003. Persistently culture positive Acanthamoeba keratitis: in vivo resistance and in vitro sensitivity. Ophthalmology 110, 1593-1600.

Pissuwan, D., Cortie, C.H., Valenzuela, S.M., Cortie, M.B., 2010. Functionalised gold nanoparticles for controlling pathogenic bacteria. Trends Biotechnol. 28, 207-213.

Raederstorff, D., Rohmer, M., 1985. Sterol biosynthesis de nova via cycloartenol by the soil amoeba Acanthamoeba polyphaga. Biochem. J. 231, 609-615.

Raederstorff, D., Rohmer, M., 1987. Sterol biosynthesis via cycloartenol and other biochemical features related to photosynthetic phyla in the amoebae Naegleria lovaniensis and Naegleria gruberi. Eur. J. Biochem 164, 427-434.

Rai, M., Deshmukh, S., Ingle, A., Gade, A., 2012. Silver nanoparticles: the powerful nanoweapon against multidrug-resistant bacteria. J. Appl. Microbiol. 112, 841-852.

Rajendran, K., Anwar, A., Khan, N.A., Aslam, Z., Raza Shah, M., Siddiqui, R., 2019. Oleic acid coated silver nanoparticles showed better in vitro amoebicidal effects against Naegleria fowleri than amphotericin B. ACS Chem. Neurosci. https://doi.org/10. 1021/acschemneuro.9b00289.

Rasmussen, B., Bush, K., Tally, F., 1997. Antimicrobial resistance in anaerobes. Clin. Infect. Dis. 24, S110-S120.

Rostom, S.A., Ashour, H.M., El Razik, H.A.A., Abd El Fattah, H., El-Din, N.N., 2009. Azole antimicrobial pharmacophore-based tetrazoles: synthesis and biological evaluation as potential antimicrobial and anticonvulsant agents. Bioorg. Med. Chem. 17, $2410-2422$.

Sadiq, S.A., Azuara-Blanco, A., Bennett, D., Lloyd, J.H., Dua, H.S., 1998. Evaluation of contamination of used disposable contact lenses by Acanthamoeba. The CLAO J.: Off. Publ. Contact Lens Associat. Ophthalmol., Inc 24, 155-158.

Sakharkar, K.R., Sakharkar, M.K., Chow, V.T., 2004. A novel genomics approach for the identification of drug targets in pathogens, with special reference to Pseudomonas aeruginosa. In Silico Biol. 4, 355-360.

Schuster, F., 1993. Comparative effects of selected azole compounds on trophic and cystic stages of Acanthamoeba polyphaga. J. Eukaryot. Microbiol. 40, 563-569.

Schuster, F.L., Guglielmo, B.J., Visvesvara, G.S., 2006. In-vitro activity of miltefosine and voriconazole on clinical isolates of free-living amebas: Balamuthia mandrillaris, Acanthamoeba spp., and Naegleria fowleri. J. Eukaryot. Microbiol. 53, 121-126.

Schuster, F.L., Visvesvara, G.S., 2004a. Free-living amoebae as opportunistic and nonopportunistic pathogens of humans and animals. Int. J. Parasitol. 34, 1001-1027.

Schuster, F.L., Visvesvara, G.S., 2004b. Opportunistic amoebae: challenges in prophylaxis and treatment. Drug Resist. Updates 7, 41-51.

Serrano-Luna, J.d.J., Cervantes-Sandoval, I., Calderón, J., Navarro-García, F., Tsutsumi, 
V., Shibayama, M., 2006. Protease activities of Acanthamoeba polyphaga and Acanthamoeba castellanii. Canad. J. Microbiol. 52, 16-23.

Siddiqui, R., Aqeel, Y., Khan, N.A., 2016. The development of drugs against Acanthamoeba infections. Antimicrob. Agents Chemother. 60, 6441-6450.

Siddiqui, R., Khan, N.A., 2012. Biology and pathogenesis of Acanthamoeba. Parasit. Vectors 5, 6 .

Siddiqui, R., Jarroll, E.L., Khan, N.A., 2010. Balamuthia mandrillaris: Role of galactose in encystment and identification of potential inhibitory targets. Exp. Parasitol. 126, $22-27$.

Singh, S., Bharti, N., Mohapatra, P.P., 2009. Chemistry and biology of synthetic and naturally occurring anti-amoebic agents. Chem. Rev. 109, 1900-1947.

Slater, C.A., Sickel, J.Z., Visvesvara, G.S., Pabico, R.C., Gaspari, A.A., 1994. Successful treatment of disseminated Acanthamoeba infection in an immunocompromised patient. New Engl. J. Med. 331, 85-87.

Stidd, D.A., Root, B., Weinand, M.E., Anton, R., 2012. Granulomatous amoebic encephalitis caused by Balamuthia mandrillaris in an immunocompetent girl. World Neurosurg. 78, 715 e717-715. e712.

Superko, H.R., Momary, K.M., Li, Y., 2012. Statins personalized. Med. Clin. 96, 123-139. Suri, S.S., Fenniri, H., Singh, B., 2007. Nanotechnology-based drug delivery systems. J. Occup. Med. Toxicol. 2, 16.

Thomson, S., Rice, C.A., Zhang, T., Edrada-Ebel, R., Henriquez, F.L., Roberts, C.W., 2017. Characterisation of sterol biosynthesis and validation of $14 \alpha$-demethylase as a drug target in Acanthamoeba. Sci. Rep. 7, 8247.
Tiewcharoen, S., Junnu, V., Chinabut, P., 2002. In vitro effect of antifungal drugs on pathogenic Naegleria spp. Southeast As. J. Trop. Med. Public Health 33, 38-41.

Tu, E.Y., Joslin, C.E., Shoff, M.E., 2010. Successful treatment of chronic stromal Acanthamoeba keratitis with oral voriconazole monotherapy. Cornea 29, 1066.

Upcroft, J.A., Campbell, R.W., Benakli, K., Upcroft, P., Vanelle, P., 1999. Efficacy of new 5-nitroimidazoles against metronidazole-susceptible and-resistant Giardia, Trichomonas, and Entamoeba spp. Antimicrob. Agents Chemother. 43, 73-76.

Walochnik, J., Duchêne, M., Seifert, K., Obwaller, A., Hottkowitz, T., Wiedermann, G. Eibl, H., Aspöck, H., 2002. Cytotoxic activities of alkylphosphocholines against clinical isolates of Acanthamoeba spp. Antimicrob. Agents Chemother 46, 695-701.

Webster, D., Umar, I., Kolyvas, G., Bilbao, J., Guiot, M.C., Duplisea, K., Qvarnstrom, Y., Visvesvara, G.S., 2012. Treatment of granulomatous amoebic encephalitis with voriconazole and miltefosine in an immunocompetent soldier. Am. J. Trop. Med. Hyg. 87, 715-718.

Wu, D., Feng, M., Wang, Z.-x., Qiao, K., Tachibana, H., Cheng, X.-j., 2018. Molecular and biochemical characterization of key enzymes in the cysteine and serine metabolic pathways of Acanthamoeba castellanii. Parasit. Vectors 11, 604

Yim, B., Park, J.-H., Jeong, H., Hong, J., Kim, M., Chang, M., Chuck, R.S., Park, C.Y., 2018. Effect of nitric oxide on Acanthamoeba castellanii. Invest. Ophthalmol. Vis. Sci. $59,3239-3248$.

Zhang, L., Pornpattananangkul, D., Hu, C.-M., Huang, C.-M., 2010. Development of nanoparticles for antimicrobial drug delivery. Curr. Med. Chem. 17, 585-594. 